\title{
Pertussis in infants, in their mothers and other contacts in Casablanca, Morocco
}

\author{
Khalid Katfy ${ }^{1,2^{*}}$, Idrissa Diawara ${ }^{1,2,6}$, Fakhredine Maaloum ${ }^{1,2}$, Siham Aziz ${ }^{2}$, Nicole Guiso ${ }^{5}$, Hassan Fellah ${ }^{3}$, \\ Bouchra Slaoui ${ }^{4}$, Khalid Zerouali, ${ }^{1,2}$, Houria Belabbes ${ }^{1,2}$ and Naima Elmdaghri ${ }^{1,2}$
}

\begin{abstract}
Background: In recent decades, there has been a marked increase in the number of reported cases of pertussis around the world, and pertussis continues to be a frequently occurring disease despite an effective childhood vaccination. This study aims to determine the role of household contacts of children diagnosed with pertussis in Casablanca Morocco.
\end{abstract}

Methods: From November 2015 to October 2017, children suspected of whooping cough that consulted Ibn Rochd University hospital at Casablanca with their household contacts were enrolled in the study. Nasopharyngeal (NP) samples of the suspected children were analyzed by culture and RT-PCR. For the household contacts, NP and blood samples were collected and analyzed by RT-PCR and specific detection of pertussis toxin antibodies by ELISA, respectively.

Results: During the study period, the survey was carried out on 128 infants hospitalized for pertussis suspicion and their families $(N=140)$. B. pertussis DNA was specifically detected in $73(57 \%)$ samples, coexistence of $B$. pertussis and B. parapertussis DNA in $3(2.3 \%)$ samples, coexistence of B. pertussis and B. holmesii DNA in $10(7.81 \%)$ and only one (0.78\%) sample was IS 481 RT-PCR positive without the possibility of determining the Bordetella species with the diagnostic tools used. Confirmations of Pertussis infection in household contacts by culture, RT- PCR and serology were 10, 46 and $39 \%$, respectively.

B. pertussis DNA was confirmed in the infants as well in their mothers in 38\% of the cases. Co detection of $B$. pertussis and B. parapertussis DNA in 2\% and co-detection of B. pertussis and B. holmesii DNA in 4\%. B. holmesii DNA alone was detected in 5 NP samples of index cases and their mothers.

Conclusions: The results of this study confirm that B. pertussis is still circulating in children and adults, and were likely a source of pertussis contamination in infants still not vaccinated. The use of RT-PCR specific for B. pertussis in the diagnosis of adults is less sensitive and should be associated with serologic tests to improve diagnosis of pertussis and contributes to preventing transmission of the disease in infants.

Keywords: Pertussis, B. holmesii, B. Parapertussis, Household contacts, Anti-PT IgG/lgA

\section{Background}

In recent decades, there has been a marked increase in the number of reported cases of pertussis around the world, and pertussis continues to be a frequently occurring disease despite an effective childhood vaccination [1]. Resurgence has been reported in many countries

\footnotetext{
* Correspondence: khalidkatfy@hotmail.com

'Department of Microbiology, Faculty of Medicine and Pharmacy, 19 rue Tarik Bnou Zyad, 20360 Casablanca, Morocco

${ }^{2}$ Bacteriology-Virology and Hospital Hygiene Laboratory, University Hospital Centre Ibn Rochd, 1, Rue des Hôpitaux, 20100 Casablanca, Morocco
} Full list of author information is available at the end of the article worldwide, even in countries with high vaccine coverage [2], probably due to multifactorial causes: atypical or non- characteristic symptomatology of classical pertussis in adolescents and adults, increased clinician awareness and reporting, contribution of sensitive biological diagnostic tools such as a sensitive and an easier real-time PCR (RT-PCR), low vaccine coverage, particularly for boosters [3, 4]. Studies of children's family with confirmed pertussis disease has attracted global concern, it is useful to detect new cases independently of the symptoms they have. Indeed, pertussis in adults has been

(C) The Author(s). 2020 Open Access This article is distributed under the terms of the Creative Commons Attribution 4.0 International License (http://creativecommons.org/licenses/by/4.0/), which permits unrestricted use, distribution, and 
reported elsewhere in several studies [5]. The prevalence of pertussis in this age group remains underestimated [6], due to the variety of clinical symptoms, the absence or differences in diagnostic methods and case definitions used. Since the introduction of vaccination in young children, a change of transmission has been observed. Adults are now shown to transmit pertussis to their unvaccinated or partly immunized infants and children [7] [8];.

Epidemiological surveillance of pertussis in household requires the adaptation of highly relevant biological diagnostic tools, in particular specific PCR able to distinguish between Bordetella species [9] [10];. Currently available $B$. pertussis diagnostic methods include direct diagnosis such as culture, specific RT-PCR and indirect diagnosis such as the detection of anti-pertussis toxin antibodies [4]. As previously reported by several authors [11], culture and RT-PCR are specific and sensitive in infants and young children but less specific in adolescents and adults due to the carriage of $B$. holmesii, and less sensitive since often adolescents and adults are coming after more than 3 weeks of cough or already treated by macrolides [12] [13];. After three weeks of cases, pertussis can be diagnosed by quantification of anti-pertussis toxin antibodies by Enzyme linked immuosorbent assay (ELISA) [4]. Serologic diagnosis in infants is very rarely useful, due to the delay in antibodies levels elevation; moreover the infant's serum may contain antibodies transmitted by the mother up to 6 months after birth [14].

In Morocco, Pertussis vaccination [pertussis whole cell vaccine $(\mathrm{wP})$ in combination with diphtheria and tetanus toxoids (DTwP)] was introduced by the national immunization program (NIP) in the early 1980's to prevent pertussis [15]. The Moroccan vaccination strategy includes a primary vaccination at 2, 3 and 4 months of age and two boosters at 18 months of age and 5 years of age [16]. The vaccine pertussis vaccine coverage exceeds $95 \%$ at the age of 24 months [17].

In Morocco, there are few epidemiological data on pertussis infection in household contacts and their involvement of pertussis diseases among children [16]. The purpose of this study was to determine the role of household contacts of children diagnosed with pertussis in Casablanca Morocco.

\section{Methods}

\section{Study design}

This cross-sectional study of whooping cough was conducted from November 2015 to October 2017 with the participation a public hospital network in GrandCasablanca - Morocco, including all patients under 14 years and child's household contacts. For each subject, the basic demographic and epidemiological data collected and recorded, such as sex, age, date of sampling, address, medical history associated with chronic diseases, and vaccination status.

The study was performed at the Microbiology Laboratory of Ibn Rochd University Hospital Centre of Casablanca (IR-UHC). In the IR-UHC, pediatric patients are managed at the Abderrahim Harrouchi Children University Hospital. All cases of serious diseases such as pertussis and complicated diseases in other hospitals are systematically transferred to IR-UHC.

\section{Laboratory methods}

Samples - Nasopharyngeal aspiration (NPA): All index cases and household contacts were sampled according to our previously published procedures [16].

- Blood sampling: Blood samples were collected in vacutainer and tested in all household contacts. The serum was separated directly or after blood sampling ( $24 \mathrm{~h}$ at room temperature). In our study, serum with hemolysis or volume less than $100 \mu \mathrm{L}$ were not used for the study. All serum were stored at $-80^{\circ} \mathrm{C}$ prior to analysis.

NP samples or blood samples taken from hospitalized patients and household contacts were sent to the microbiology laboratory of Ibn Rochd University Hospital in Casablanca at room temperature, accompanied by a fact sheet with all clinical and socio-demographic indications.

Mothers were automatically sampled with their children. Other members of the family have benefited of $\mathrm{NP} /$ serum samples only when the index case is positive and these household contacts have compatible signs such as prolonged cough.

Duplicates of the same person are excluded from the analysis.

Direct diagnosis a) As for the direct diagnosis, we used our previously methods [16] for the bacterial culture of Bordetella species and real-time PCR for the detection of the presence of Bordetella strains harboring IS481 (Bordetella spp), IS1001 (B. parapertussis), ptxA-Pr (B. pertussis) and h-IS1001 (B. holmesii).

b) Indirect diagnosis methods: Serology by ELISA (Enzyme Linked Immunosorbent assays).

In this study, measurement of antibodies to B. pertussis antigens was done by ELISA). Serum samples were analyzed by using a commercial kit (SeroPertussis ${ }^{\mathrm{ma}}$ Toxin IgA Kit and SeroPertussis ${ }^{\mathrm{Tu}}$ Toxin IgG Kit -Savyon - Diagnostics Ltd) used for quantitative detection of IgG and IgA antibodies to Pertussis Toxin in human serum, and expressed in International Units per milliliter $(\mathrm{IU} / \mathrm{ml})$. The microplates were read on the 'BIO-RAD PR2100 Microplate Reader' (manufacturer) instrument, 
designed to measure the optical density (OD) of fluid samples in 96 well microplates [18].

The interpretation of IgG-anti-PT antibodies was as recommended by manufacturer (Fig. 1). The IgG-anti-PT values below to $40 \mathrm{IU} / \mathrm{ml}$ were not indicator of recent contact [19] [20]. Whereas levels above $>100 \mathrm{IU} / \mathrm{ml}$ can be used as an indicator of recent contact with the bacteria. If diagnosis cannot be established with certainty (Single serum, Intermediate range), Savyon Diagnostics recommends testing IgA levels, which may serve as an additional test for equivocal results ( $>40$ and $<100 \mathrm{IU} / \mathrm{ml}$ ).

\section{Data management and statistics}

Data entry was performed using WHONET 5.6 software. Analyzes were performed using Epi info (CDC, Atlanta, Georgia), Microsoft Excel and the JASP software [21], a cross platform statistical software program with a stateof-the-art graphical user interface.

The statistical significance adopted for the study was $5 \%$ ( $p$-values $<0.05)$.

\section{Results}

During the study period, between November 2015 and October 2017, 268 NP samples were collected from infants consulted Ibn Rochd University hospital at Casablanca with clinical suspicion of pertussis disease and some of their household contacts.

Hospitalized children were below 5 years of age, with an average of $60 \pm 10$ days and $87 \%(111 / 128)$ were under 2 months of age. Household contacts were essentially mothers $(87 \%)$. The participation of other family members was very low with only 4 (3\%) fathers, 9 (6\%) brothers and siblings (five sisters, four brothers), and 5 (4\%) grandparents (Fig. 2). Eighty-two percent of the NP samples included in this study were from infants admitted to Abderrahim Harrouchi pediatric hospital. The other Casablanca hospitals were poorly represented.
Clinically, $85.6 \%$ of patients diagnosed for pertussis in this study had common symptoms of typical pertussis, $14.3 \%$ of pneumo-pertussis, sometimes complicated with superinfection in $12 \%$ of cases, syncopal apnea in $6 \%$, and cyanosis in 36\%. Administration of antibiotics, mainly macrolide family, was noted in 82 from 87 (95\%) infants diagnosed (Table 1). More than $65 \%$ of household contacts had no symptoms of whooping cough, $20 \%$ more than three weeks and $15 \%$ less than three weeks. Of these, $67 \%$ mothers had no symptoms of pertussis but were in contact with at least one person who was coughing at home.

Cultures of NP samples were performed from 51 of the 128 index cases and from 51 of the 140 contacts, other NP samples were not tested by culture for various reasons e.g., unavailability of culture media, samples improperly transported or stored. The culture was positive in $16 \%(8 / 51)$ of the index cases and $10 \%(5 / 51)$ of the contacts.

All samples were tested by RT-PCR, Among 128 NPA of infants included, IS 481 RT-PCR was positive in $68 \%$ $(87 / 128)$, the majority was observed in unvaccinated children less than 2 months of age $74 \%$ (64/87). Other incompletely vaccinated $14 \%(12 / 87)$ with one or two doses were aged between 3 and 14 months. B. pertussis DNA was specifically detected in 73 (57\%) samples, coexistence of B. pertussis and B. parapertussis DNA in 3 (2.3\%) samples, coexistence of B. pertussis and B. holmesii DNA in $10(7.81 \%)$ and only one $(0.78 \%)$ sample was IS 481 RT-PCR positive without the possibility of determining the Bordetella species with the diagnostic tools used. Six NPA were positive only for B. holmesii DNA only. Bordetella DNA was not detected in 41 (32\%) samples. No co-infection between $B$. parapertussis and $B$. holmesii was found (Fig. 2).

No Bordetella DNA was detected in the NPA of the household members of the non-infected infants. Among

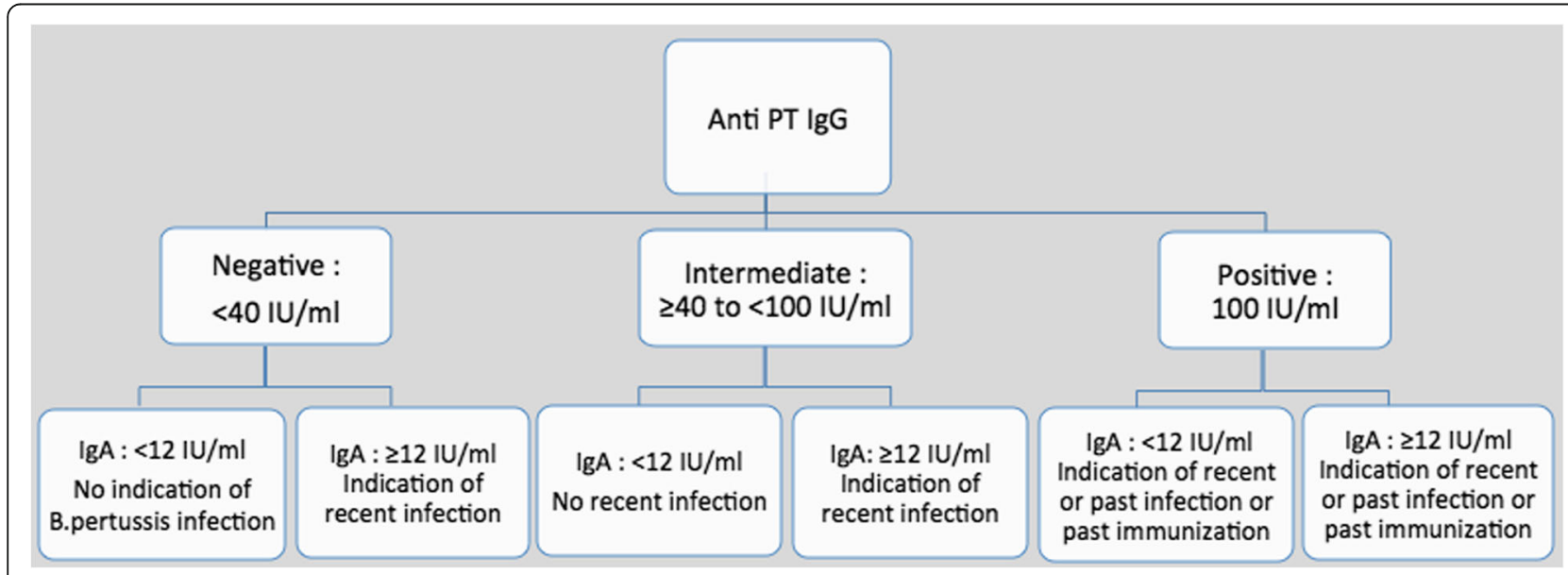

Fig. 1 Interpretation of the results according to the $\operatorname{lgG} / \mathrm{lgA}$ antibody profile in patient serum [19] 


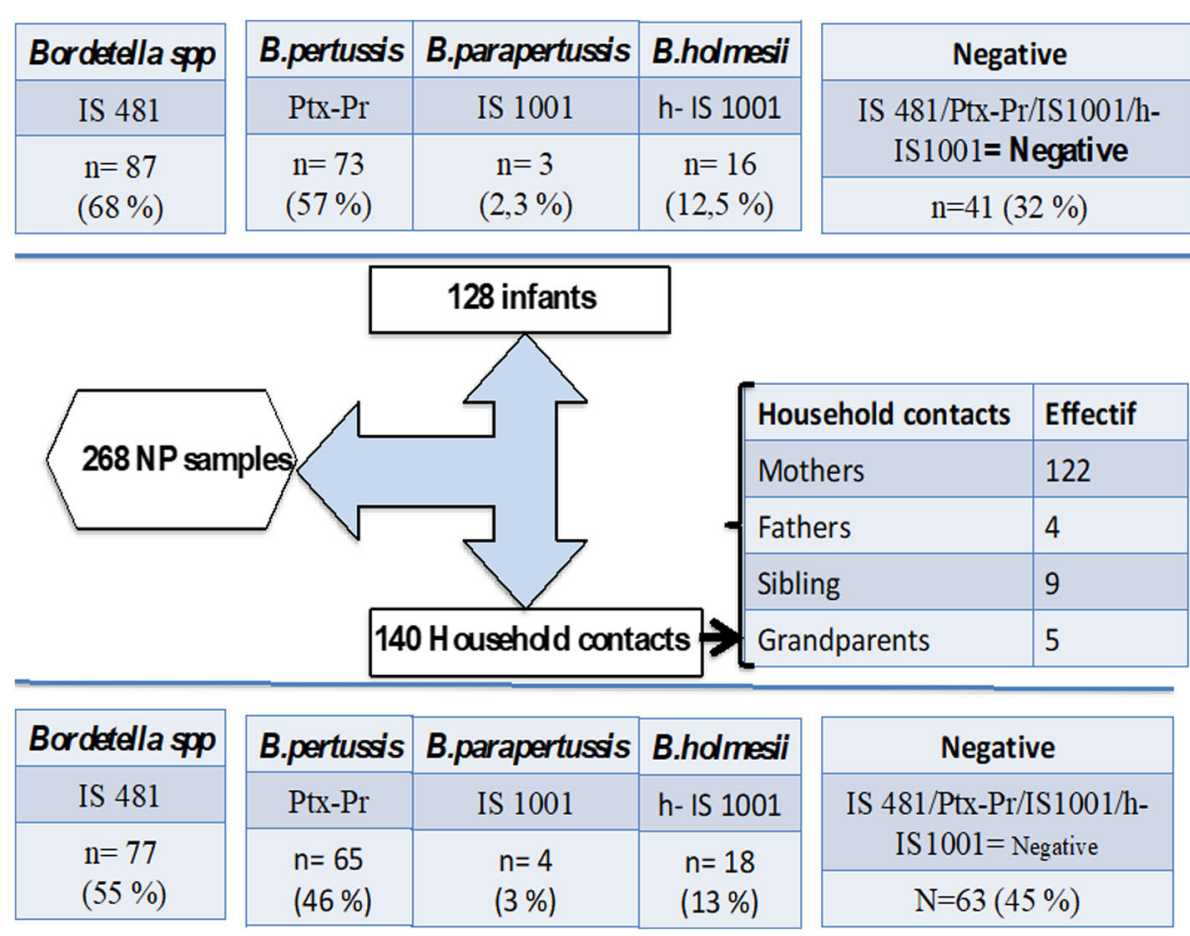

Fig. 2 Results of confirming the diagnosis of pertussis in infants and household contacts

the 140 NPs of household contacts, IS 481 RT-PCR was detected in $55 \%(77 / 140)$. B. pertussis DNA in $46 \%(65 /$ $140)$ samples, $B$. parapertussis in $3 \%(4 / 140)$ samples, $B$. holmesii in 13\% (18/140) samples, and three NPs were positive by RT-PCR IS 481 alone and identified as Bordetella spp. The coexistence of B. pertussis and B. parapertussis DNA was detected in $3(2 \%)$ samples, $B$. pertussis and $B$. holmesii in $6(4 \%)$ samples. No co-infection between B. parapertussis and B. holmesii DNA was found. $B$. holmesii DNA was detected in 6 index cases and 5 of their mothers. Pertussis was confirmed by RT-PCR IS 481 in 64 from 122 (52\%) mothers. Also other family members participating in this survey were not spared from the infection: 3 of 5 grandparents, 8 of 9 siblings and one of 4 fathers were also pertussis positive. Pertussis was confirmed in children and their mothers together by PCR for Bordetella spp in $50 \%(61 / 122)$, B. pertussis in $40 \%$ (49/ $122)$, and B. holmesii in $8 \%(10 / 122)$. No B. parapertussis was detected (Fig. 2). Of these, only 32 from 49 mothers were confirmed serologically (Figs. 3 and 4).

Table 1 Clinical information of patients and their household contacts

\begin{tabular}{|c|c|c|c|c|c|c|}
\hline & \multicolumn{2}{|l|}{ Infants diagnosed } & \multicolumn{4}{|l|}{ Household contacts } \\
\hline & Not confirmed $(n=41)$ & Confirmed $(n=87)$ & Mothers $(n=122)$ & Fathers $(n=4)$ & Siblings $(n=9)$ & Grandparents $(n=5)$ \\
\hline \multicolumn{7}{|l|}{ Symptoms } \\
\hline Coughingparoxysm & $18(44 \%)$ & $40(46 \%)$ & $10(8 \%)$ & $0(0 \%)$ & $2(22 \%)$ & $1(20 \%)$ \\
\hline Coughing & $21(51 \%)$ & $60(69 \%)$ & $45(37 \%)$ & $4(100 \%)$ & $6(67 \%)$ & $4(80 \%)$ \\
\hline Singing Rooster & $27(66 \%)$ & $74(85 \%)$ & $1(0,8 \%)$ & $0(0 \%)$ & $1(11 \%)$ & $0(0 \%)$ \\
\hline Vomiting & $23(56 \%)$ & $9(10 \%)$ & $9(7 \%)$ & $0(0 \%)$ & $3(33 \%)$ & $2(40 \%)$ \\
\hline Cyanosis & 20 (49\%) & $30(34 \%)$ & $0(0 \%)$ & $0(0 \%)$ & $0(0 \%)$ & $0(0 \%)$ \\
\hline Apnea & $4(10 \%)$ & $5(6 \%)$ & $0(0 \%)$ & $0(0 \%)$ & $0(0 \%)$ & $0(0 \%)$ \\
\hline Respiratory distress & $23(56 \%)$ & $26(30 \%)$ & $37(30 \%)$ & $4(100 \%)$ & 7 (78\%) & $4(80 \%)$ \\
\hline \multicolumn{7}{|l|}{ Antibiotictreatment } \\
\hline Yes & $37(89,7 \%)$ & $82(94,7 \%)$ & $8(6,9 \%)$ & $4(100 \%)$ & $2(22 \%)$ & $2(40 \%)$ \\
\hline No & $4(10,2 \%)$ & $05(5,2 \%)$ & $114(93 \%)$ & $0(0 \%)$ & $2(22 \%)$ & $1(20 \%)$ \\
\hline Hospitalization & 37 (91\%) & 87 (100\%) & $0(0 \%)$ & $0(0 \%)$ & $1(11 \%)$ & $0(0 \%)$ \\
\hline
\end{tabular}



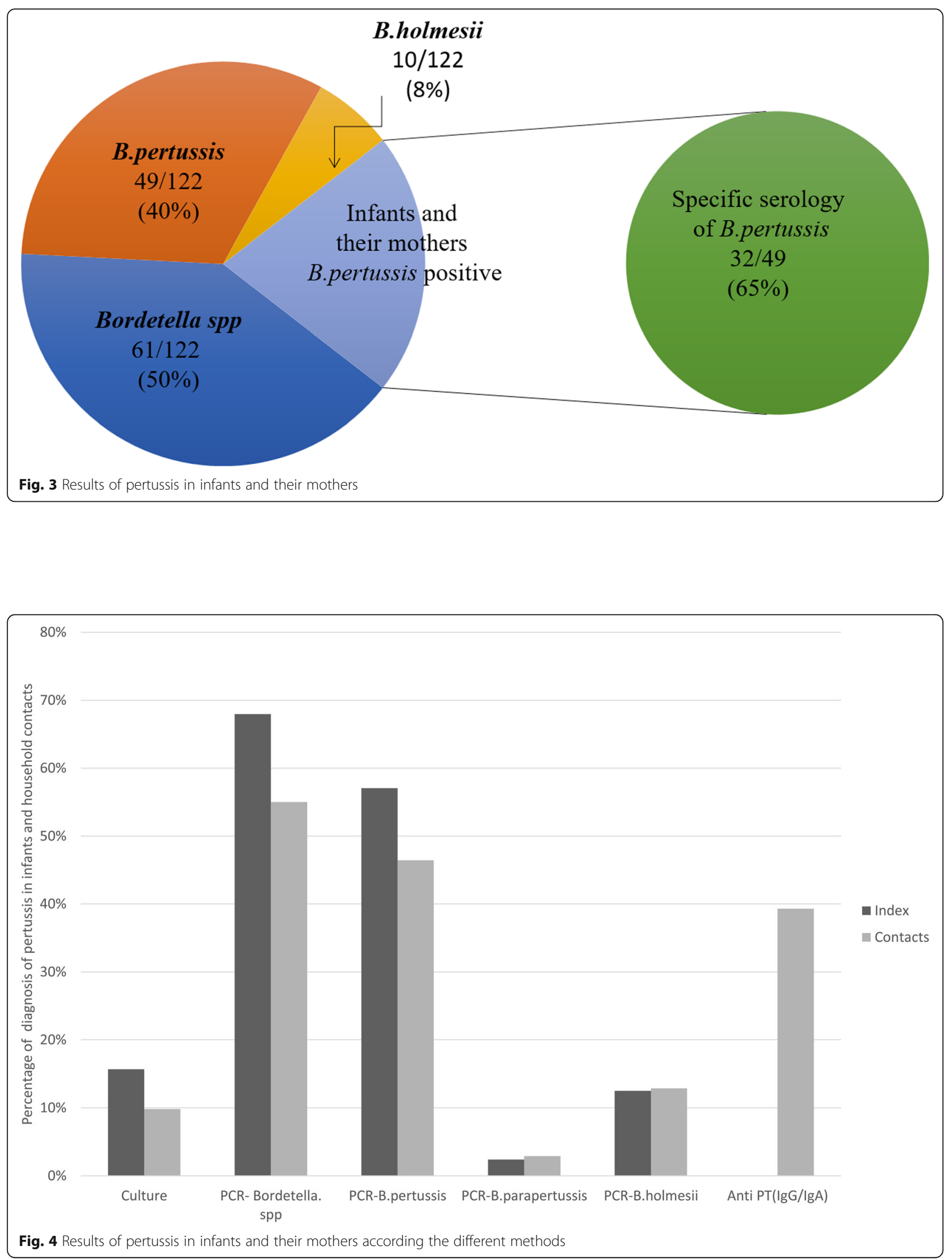
A total of 140 blood serum were analyzed only in household contacts with single serum because of parents refusal to return for a second blood test or technical difficulties in getting a venous blood sample. Serology confirmed infections in 55 from 140 (39\%) household contacts by anti-PT (IgG and IgA) antibodies tests. Over $100 \mathrm{IU} / \mathrm{ml}$ of anti-PT IgG were measured in 5 serums and interpreted as an indicator of recent contact with the bacterium. Between 40 and $100 \mathrm{UI} / \mathrm{ml}$ in 17 serums, to analyze this intermediate results depend of Antipertussis IgA antibodies tests, 12 of them were considered as a recent $B$. pertussis infection. Anti-PT IgG $>40$ and IgA $>12$ in 15 cases, and anti-PT IgA $>12 \mathrm{UI} / \mathrm{ml}$ in 53 serums (Table 2).

Comparison of the serology and other diagnosis tools showed that all culture-positive were also positive by serology, and showed significantly higher levels of IgG (> $100 \mathrm{IU} / \mathrm{ml}$ ) compared to negative cultures samples.

B. pertussis infection was confirmed by real time PCR and detection of anti-PT antibodies in $41(29 \%)$ cases and only by RT-PCR in 22 (16\%) of cases. In 12 (9\%) cases, $B$. pertussis infection was confirmed only by detection of anti-PT antibodies in the serum of the patients. $B$. holmesii was found in 16 index cases and in 10 of their mothers, 4 of them were co-infected with B. pertussis, these mothers were serology negative (Table 3 ).

\section{Discussion}

This study completes a previous study [16]. In the present study, we analyzed the household contacts with or without symptoms of whooping cough to determine their involvement in infant's pertussis infection. The case definition of pertussis in adolescents and adults is based solely on clinical diagnosis in Morocco. However, experts underline that current clinical case definitions cannot universally be applicable, and that different age groups should be evaluated by different clinical criteria [22] [23]. Laboratory confirmation of $B$. pertussis infection is not routinely used; therefore the rate of $B$. pertussis/B. parapertussis infections is probably underestimated. Direct and indirect laboratory methods used in this study for pertussis diagnosis are available. Direct tests are (RT-PCR) and culture, whereas indirect tests measure specific anti-PT antibodies. The choice of the biological test depends on the age and the duration of symptoms: in neonates and young infants post-onset of symptoms, PCR and/or culture should be performed. Measuring antibodies to B. pertussis antigens is mainly meaningful on household members (vaccinated older children, adolescents and adults). In cases with less than two weeks of coughing, culture and PCR from nasopharyngeal samples should be done. If coughing lasted at least 2 to 3 weeks, the measurement of IgG-anti-PT should be sufficient [20].

During the study period, every infant diagnosed was mostly accompanied by his/her mother, and very few other household members very few people were able to participate. In this study, 51 infants and their 51 contacts benefited from culture. Pertussis was faintly confirmed in 8 infants and 5 mothers. Several studies have demonstrated low culture sensitivity to confirm a case of pertussis as compared to RT-PCR and blood tests [7]. The sensitivity of cultures in pertussis diagnosis depends on the duration of symptoms, age, antibiotics treatment and the vaccination status of the patient [24]. Despite the difficulties, culture remains the "gold standard" pertussis diagnosis method due to its high specificity, and is important for following the evolution of the bacteria, and for monitoring antibiotic sensitivity especially to macrolides [25].

The performance of PCR based exclusively on IS481 is highly sensitive and confirmed the circulation of Bordetella spp in 87 (68\%) infants and $77(55 \%)$ in their household contacts respectively. It was also reported that other Bordetella species sometimes associated with respiratory disease in humans, including $B$. bronchiseptica and $B$. holmesii harboring the IS481 sequence, which may lead to misidentification as B. pertussis [26] [27]. However, to differentiate Bordetella species and to avoid false positive results, we used other targets specific for $B$. pertussis, B. parapertussis and B. holmesii [28]. Our results showed the predominance of $B$. pertussis among NP samples from 73 (57\%) infants and $65(46 \%)$ of household members, it should be noted that $87 \%$ (76 of 87) were infants less than two months of age and didn't

Table 2 Results of interpretation of the serological profiles

\begin{tabular}{|c|c|c|c|c|}
\hline \multicolumn{5}{|c|}{$\lg G$ and $\lg A$ interpretation } \\
\hline IgG results & $\begin{array}{l}\lg A \\
\text { results }\end{array}$ & $\begin{array}{l}\text { Indication of a recent B.pertussis infection, or } \\
\text { recently vaccinated }\end{array}$ & $\begin{array}{l}\text { Absence of recent infection, or past infection, or } \\
\text { past immunization }\end{array}$ & $\begin{array}{l}\text { Recent contact with the } \\
\text { bacterium }\end{array}$ \\
\hline \multirow[t]{2}{*}{ Intermediate } & Positive & 12 & 0 & 0 \\
\hline & Negative & 0 & 0 & 5 \\
\hline \multirow{2}{*}{$\begin{array}{l}\text { Highly } \\
\text { Positive }\end{array}$} & Positive & 3 & 0 & 0 \\
\hline & Negative & 2 & 0 & 0 \\
\hline \multirow[t]{2}{*}{ Negative } & Positive & 38 & 0 & 0 \\
\hline & Negative & 0 & 80 & 0 \\
\hline
\end{tabular}


Table 3 Contribution of RT-PCR/ anti-PT antibodies tests for diagnosis pertussis in household contacts

\begin{tabular}{llll}
\hline $\lg$ \& IgA anti-PT & \multicolumn{1}{l}{ RT-PCR Ptx-Pr Positive in household contacts } & Positive & Total \\
\cline { 2 - 4 } & Negative & 43 & 55 \\
\hline Indication of recent infection & 12 & 18 & 80 \\
No indication of B. pertussis infection & 62 & 4 & 5 \\
Recent contact with B. pertussis & 1 & 65 & 140 \\
Total & 75 & 5 & \\
\hline
\end{tabular}

receive any dose of vaccines according to the Moroccan vaccine strategy recommendation, leaving these children in danger of direct contamination. B. parapertussis was detected in 3 infants samples as well in the household contacts. In these infants samples, $B$. pertussis was also detected. B. parapertussis remains low compared to neighboring countries [29].

B. holmesii was detected in $16 \mathrm{NP}$ samples from index cases and 18 of their contacts. Coexistence of $B$. holmesii and $B$. pertussis was observed in 10 cases with $B$. pertussis, as observed in other studies $[9,10,16,18]$.. In 6 index cases and 8 household contacts, unfortunately no other microorganisms were searched. For this reason we couldn't determine whether $B$. holmesii was responsible of the cough or not. Coexistence of $B$ holmesii and $B$. parapertussis was not observed in this study.

We retained no difference in the demographics, clinical features, and vaccination status among patients infected by $B$. holmesii and B. pertussis, as reported previously [9].

RT-PCR provides a sensitive and specific diagnosis of B. pertussis infections in infected cases for a period not exceeding 3 to 4 weeks maximum, beyond that, it will be negative [20]. For this reason for adolescents or adults coughing more than 3 weeks serology can be used.

In this study, anti-PT IgG concentrations were measured using a commercial kit, comparable to other serological kits tested previously [19]. This kit was reported to have a sensitivity and specificity up to 88 and $100 \%$, respectively [18]. Whereas other techniques, such as agglutination, indirect fluorescence, immunoblotting or complement fixation, are discouraged [30]. If diagnosis cannot be established with certainty, or in case of nonavailability of a second serum sample, we used a second commercial kit $[19,20]$. This test utilizes purified pertussis toxin as an antigen, allowing quantitative determination of IgA antibodies to Pertussis Toxin according to the first International WHO Standard [31].

Previously, there were opinions that eliminated the measurement of $\operatorname{IgA}$ in the serological diagnosis of pertussis [32, 33]. However, a European collaboration [20], suggested that IgA antibodies have a marginal value for the serological diagnosis of pertussis and can be used as an additional method only for testing serum with antiPT IgG concentrations in ranges that were undetermined which facilitate results interpretation.
Anti-PT IgG / IgA antibodies was confirmed in 55 (39\%) cases indicating for an acute infection or recent contact. B. pertussis was confirmed by both biological tests (PCR and anti-PT antibodies) in 41 (29\%) household members. Twelve (9\%) cases were confirmed only by serological tests. These results showed the contribution of serological test to identify some cases that can be misinterpreted as false negatives, especially in people who had delayed their diagnosis or had prolonged cough, those data are similar to those reported by previous studies [18].

A total of $16 \%(22 / 140)$ of the household contacts had anti-PT IgG levels superior than or equal to $40 \mathrm{IU} / \mathrm{mL}$, including 5 cases with anti-PT IgG antibodies superior than or equal to $100 \mathrm{IU} / \mathrm{mL}$ and interpreted as an indication of recent $B$. pertussis infection, usually seen in cases diagnosed at the onset of cough, or recently vaccinated. These results are consistent with the previous kinetics of antibody titers after infection: kinetics differ according to whether patients never was in contact with the bacteria before infection or vaccinated or previously infected [34]. 77\% (17/22) cases had intermediate levels of anti-PT IgG ( $>40$ and $<100 \mathrm{IU} / \mathrm{mL}$ ), indicating possible infection.

Among these 17 suspected IgG tests, 70\% (12/17) had IgA titers indicating a recent infection. The other 5 cases had an IgA value of strictly less than $12 \mathrm{IU} / \mathrm{ml}$, and interpreted as the absence of recent infection or past infection or past immunization. Then, when its possible the addition of IgA measurement might be useful.

In our results we detected presence of $B$. holmesii and B. parapertussis DNA in NPs from adults. Serology cannot identify these species. Numerous studies have shown that other Bordetella species can be the source of infection / carriage in adults. This leads to propose the development of other serological kits intended for the detection of emergent species of Bordetella.

Our results showed that B. pertussis was detected in 77 (55\%) symptomatic household members, 53 (38\%) from mothers, 8 (6\%) from siblings, 3 (2\%) from grandparents and only one from father and in 47 (64\%) hospitalized infants and their mothers. This result suggests that mothers were largely the source of the infection and transmitted the disease to their children, this finding confirms other reports [35]. However, in our study, mothers who brought 
the child to the hospital, were the contaminator in the majority of cases (86\%). These mothers have been systematically sampled, when their children were declared as having whooping cough. This could distorts statistical comparisons between household members. Furthermore, we focused mainly on symptomatic case while pertussis case contact could be asymptomatic. Another limitation in the study was about the serological analysis. Indeed, it's possible to have a high rates of false positives when the diagnosis is basis on serology. When pertussis diagnosis is based on positive serology, there are need to know that the persons were not immunized in the last year because elevated IgG can be result of their vaccination. These information were not explore in our study. We found that in 19 confirmed infants, mothers were not infected by $B$. pertussis, indicating that mothers were not the only possible source of contamination. The contaminator could have been another household members as father, sibling or grandparents [5] [36] [37] [38]. In the US, where whole-cell vaccine is used, a study indicate that the source of infant pertussis has shifted from the mother to the adolescent siblings [39]. Continued monitoring of the source of infant pertussis through surveillance is important, especially as the epidemiology of pertussis changes over the time.

\section{Conclusion}

In conclusion, the results of this study suggest that, despite a high vaccination coverage rate of $95 \%$ in primary vaccination in Casablanca, pertussis is not controlled and dangerously present in household contacts of infants. A rapid diagnosis of pertussis in infants using RT-PCR is of high importance in order to treat with macrolides all persons around the infant in order to stop transmission of the disease. Unveiling household contacts pertussis contaminated by RT-PCR alone is less sensitive, especially in who coughed for a long time, it must be completed if it's negative by serologic diagnosis. This study is the basis of a perennial surveillance in Morocco, not only in Casablanca but in the whole country. Increasing awareness of pertussis among General Practitioners, health care workers but also the Public is also a major objective in our country.

\section{Abbreviations}

ELISA: Enzyme linked immuosorbent assay; IR-UHC: Ibn Rochd University Hospital Centre of Casablanca; NPA: Nasopharyngeal aspiration; ptx: pertussis toxin gene; RT-PCR: real time polymerase chain reaction; WHO: World Health Organization

\footnotetext{
Acknowledgements

We would like to thank the study participants for their contribution to this study. We Gratefully acknowledge Dr. S.GUILLOT and colleagues of the French National Reference Center of Whooping Cough and Other Bordetelloses at the Institute Pasteur of Paris for provision of laboratory protocols and for providing reference strains. We also thank Dr. N. GAOUGAOU for their technical assistance and great support throughout this study.
}

Conflict of interest

The authors declare that they have no conflicts of interest.

\section{Authors' contributions}

$\mathrm{NE}, \mathrm{KK}, \mathrm{KZ}, \mathrm{ID}$ and NG conceived and designed the study. KK, BS, HB, SA and FM conducted the case surveillance and collected clinical data. ID, FM and KK conducted the laboratory assays. NG, HF, NE, ID and KK analyzed the data. $N G, N E, K K, I D$ and KZ drafted the manuscript. All authors read and approved the final manuscript

\section{Funding}

This work was supported by an unrestricted grant of SANOFI-PASTEUR laboratory. The authors conceived the study, and the study design was developed and agreed to by the authors without any input from the funding body. The funding body was not involved in and, had no influence over, study design, data collection, data analyses, interpretation of results, report writing or in the decision to submit the paper for publication.

\section{Availability of data and materials}

All the data supporting the conclusions of the present study are included within the manuscript. Supplementary datasets used and/or analyzed during the current study are available from the corresponding author on reasonable request.

\section{Ethics approval and consent to participate}

The study proposal was reviewed and approved by the Institutional Review Board of the University Mohammed V - Soussi, Faculty of Medicine, Pharmacy and Dental Medicine of Rabat, Morocco N77/16. The study was anonymous and all patients and household contacts, and their parents/ guardians for minors below 18 years of age, were informed by their physicians about the nature of the study. Only patients or theirs parents/ guardians for minors who agreed to participate in this study signed the informed consent. The fact that samples will be stored for a certain period of time before testing was clearly indicated in the inform consent statement.

\section{Consent for publication}

All authors, participants (The patients/legal guardians) and partners agreed the publication of data presented in this manuscript

\section{Author details}

'Department of Microbiology, Faculty of Medicine and Pharmacy, 19 rue Tarik Bnou Zyad, 20360 Casablanca, Morocco. ${ }^{2}$ Bacteriology-Virology and Hospital Hygiene Laboratory, University Hospital Centre Ibn Rochd, 1, Rue des Hôpitaux, 20100 Casablanca, Morocco. ${ }^{3}$ Department of Immunology, Faculty of Medicine and Pharmacy, 19 rue Tarik Bnou Zyad, 20360 Casablanca, Morocco. ${ }^{4}$ Abderrahim Harouchi Pediatric Hospital, rue Mohamed El Faidouzi, -ex Jenner Quartier: Hôpitaux -, Casablanca, Morocco. ${ }^{5}$ Molecular Prevention and Therapy of Human Diseases, Institut Pasteur, 25 rue du Dr Roux, 75015 Paris, France. ${ }^{6}$ Faculté des Sciences et Techniques de Santé, Mohammed VI University of Health Sciences (UM6SS), Casablanca, Morocco.

Received: 30 April 2019 Accepted: 29 November 2019 Published online: 14 January 2020

\section{References}

1. Hartzell JD, Blaylock JM. Whooping cough in 2014 and beyond: an update and review. Chest. 2014;146:205-14.

2. Bennett JE, Dolin R, Blaser MJ, editors. Mandell, Douglas, and Bennett's principles and practice of infectious diseases. 8th ed. Philadelphia: Elsevier/ Saunders; 2015.

3. Cherry JD. Epidemic pertussis in 2012 - the resurgence of a vaccinepreventable disease. N Engl J Med. 2012;367:785-7.

4. Organization WH. Laboratory Manual for the diagnosis of whooping cough caused by bordetella pertussis. http://www.who.int/iris/handle/10665/127891 (2014). Accessed 4 Jan 2018.

5. Godoy P, García-Cenoz M, Toledo D, Carmona G, Caylà JA, et al. Factors influencing the spread of pertussis in households: a prospective study, Catalonia and Navarre, Spain, 2012 to 2013. Eurosurveillance; 21. Epub ahead of print 10 November 2016. https://doi.org/10.2807/1560-7917.ES 2016.21.45.30393. 
6. Miyashita N, Akaike H, Teranishi H, Kawai Y, Ouchi K, et al. Diagnostic value of symptoms and laboratory data for pertussis in adolescent and adult patients. BMC Infect Dis. 2013;13:129.

7. Wiley KE, Zuo Y, Macartney KK, McIntyre PB. Sources of pertussis infection in young infants: a review of key evidence informing targeting of the cocoon strategy. Vaccine. 2013;31:618-25.

8. Skoff TH, Kenyon C, Cocoros N, Liko J, Miller L, et al. Sources of infant pertussis infection in the United States. Pediatrics. 2015;136:635-41. peds. 2015-1120

9. Njamkepo E, Bonacorsi S, Debruyne M, Gibaud SA, Guillot S, et al. Significant finding of Bordetella holmesii DNA in nasopharyngeal samples from French patients with suspected pertussis. J Clin Microbiol. 2011;49:4347-8.

10. Rodgers L, Martin SW, Cohn A, Budd J, Marcon M, et al. Epidemiologic and laboratory features of a large outbreak of pertussis-like illnesses associated with cocirculating Bordetella holmesii and Bordetella pertussis--Ohio, 20102011. Clin Infect Dis Off Publ Infect Dis Soc Am. 2013;56:322-31.

11. Nakamura Y, Kamachi K, Toyoizumi-Ajisaka H, Otsuka N, Saito R, et al. Marked difference between adults and children in Bordetella pertussis DNA load in nasopharyngeal swabs. Clin Microbiol Infect Off Publ Eur Soc Clin Microbiol Infect Dis. 2011;17:365-70.

12. Riffelmann M, Wirsing von König CH, Caro V, Guiso N, Pertussis PCR Consesus Group. Nucleic acid amplification tests for diagnosis of Bordetella infections. J Clin Microbiol. 2005;43:4925-9.

13. Guidance and protocol for the use of real-time PCR in laboratory diagnosis of human infection with Bordetella pertussis or Bordetella parapertussis. European Centre for Disease Prevention and Control. http://ecdc.europa.eu/ en/publications-data/guidance-and-protocol-use-real-time-pcr-laboratorydiagnosis-human-infection (2012). Accessed 8 Jan 2018.

14. Jõgi $P$, Oona M, Toompere $K$, Lutsar I. Estimated and reported incidence of pertussis in Estonian adults: a seroepidemiological study. Vaccine. 2015;33: 4756-61.

15. WHO. Revised guidance on the choice of pertussis vaccines: July 2014. Wkly Epidemiol Rec. 2014;89:337-40. [PubMed].

16. Katfy K, Guiso N, Diawara I, Zerouali K, Slaoui B, et al. Epidemiology of pertussis in Casablanca (Morocco): contribution of conventional and molecular diagnosis tools. BMC Infect Dis. 2017:17:348.

17. Ouardirhi A. Vaccination contre la coqueluche: Le moyen de prévention le plus efficace. Albayane; 2012.

18. Dinu S, Guillot S, Dragomirescu CC, Brun D, Lazăr \$̧, et al. Whooping cough in South-East Romania: a 1-year study. Diagn Microbiol Infect Dis. 2014;78:302-6.

19. Riffelmann $M$, Thiel $K$, Schmetz J, Wirsing von Koenig CH. Performance of commercial enzyme-linked Immunosorbent assays for detection of antibodies to Bordetella pertussis. J Clin Microbiol. 2010;48:4459-63.

20. Guiso N, Berbers G, Fry NK, He Q, Riffelmann M, et al. What to do and what not to do in serological diagnosis of pertussis: recommendations from EU reference laboratories. Eur J Clin Microbiol Infect Dis Off Publ Eur Soc Clin Microbiol. 2011;30:307-12.

21. JASP Team. 2016 JASP (version 0.8)[computer software]. See https://jaspstats.org/

22. Cherry JD, Tan T, Wirsing von König C-H, Forsyth KD, Thisyakorn U, et al. Clinical definitions of pertussis: summary of a global pertussis initiative roundtable meeting, February 2011. Clin Infect Dis Off Publ Infect Dis Soc Am. 2012;54:1756-64.

23. Guiso N, Wirsing Von Koenig CH. Surveillance of pertussis: methods and implementation. Expert Rev Anti-Infect Ther. 2016;14(7):657-67. https://doi. org/10.1080/14787210.2016.1190272.

24. Castillo ME, Bada C, Del Aguila O, Petrozzi-Helasvuo V, Casabona-Ore V, et al. Detection of Bordetella pertussis using a PCR test in infants younger than one year old hospitalized with whooping cough in five Peruvian hospitals. Int J Infect Dis IJID Off Publ Int Soc Infect Dis. 2015;41:36-41.

25. Baptista PN, Magalhães VS, Rodrigues LC. The role of adults in household outbreaks of pertussis. Int J Infect Dis IJID Off Publ Int Soc Infect Dis. 2010;14:e111-4.

26. Reischl U, Lehn N, Sanden GN, Loeffelholz MJ. Real-Time PCR assay targeting IS481 of Bordetella pertussis and molecular basis for detecting Bordetella holmesii. J Clin Microbiol. 2001;39:1963-6.

27. Tizolova A, Guiso N, Guillot S. Insertion sequences shared by Bordetella species and implications for the biological diagnosis of pertussis syndrome. Eur J Clin Microbiol Infect Dis Off Publ Eur Soc Clin Microbiol. 2013;32:89-96.
28. Tatti KM, Sparks KN, Boney KO, Tondella ML. Novel multitarget real-time PCR assay for rapid detection of Bordetella species in clinical specimens. J Clin Microbiol. 2011;49:4059-66.

29. Ben Fraj I, Smaoui H, Guillot C, Bouchez V, Brisse S, Guiso N, Kechrid A Pertussis epidemiology in Tunisian infants and children and characterization of Bordetella pertussis isolates: results of a 9-year surveillance study, 2007 to 2016. J. Med Microbiol. 2019;68:241-7.

30. Kapasi A, Meade BD, Plikaytis B, Pawloski L, Martin MD, et al. Comparative study of different sources of pertussis toxin (PT) as coating antigens in lgG anti-PT enzyme-linked Immunosorbent assays. Clin Vaccine Immunol CVI. 2012;19:64-72.

31. WHO International Standard Pertussis Antiserum (Human) 1st IS NIBSC code: 06/140 Instructions for use (Version 2.0, Dated 09/04/2013). http://www. nibsc.org/documents/ifu/06-140.pdf. Accessed 9 Jan 2018.

32. Mattoo S, Cherry JD. Molecular pathogenesis, epidemiology, and clinical manifestations of respiratory infections due to Bordetella pertussis and other Bordetella subspecies. Clin Microbiol Rev. 2005;18:326-82.

33. Ward JI, Cherry JD, Chang S-J, Partridge S, Keitel W, et al. Bordetella pertussis infections in vaccinated and unvaccinated adolescents and adults, as assessed in a national prospective randomized Acellular Pertussis Vaccine Trial (APERT). Clin Infect Dis Off Publ Infect Dis Soc Am. 2006;43:151-7.

34. Storsaeter J, Hallander HO, Gustaffson L, Olin P. Levels of anti-pertussis antibodies related to protection after household exposure to Bordetella pertussis. Vaccine. 1998;16:1907.

35. Bisgard KM, Pascual FB, Ehresmann KR, Miller CA, Cianfrini C, et al. Infant pertussis: who was the source? Pediatr Infect Dis J. 2004:23:985-9.

36. Elumogo TN, Booth D, Enoch DA, Kuppuswamy A, Tremlett C, et al. Bordetella pertussis in a neonatal intensive care unit: identification of the mother as the likely source. J Hosp Infect. 2012;82:133-5.

37. de Greeff SC, de Melker HE, Westerhof A, Schellekens JFP, Mooi FR, et al. Estimation of household transmission rates of pertussis and the effect of cocooning vaccination strategies on infant pertussis. Epidemiol Camb Mass. 2012;23:852-60.

38. Baxter R, Bartlett J, Rowhani-Rahbar A, Fireman B, Klein NP. Effectiveness of pertussis vaccines for adolescents and adults: case-control study. BMJ. 2013; 347:f4249.

39. Skoff TH, Kenyon C, Cocoros N, Liko J, Miller L, et al. Sources of infant pertussis infection in the United States. Pediatrics. 2015;136(4):635-41.

\section{Publisher's Note}

Springer Nature remains neutral with regard to jurisdictional claims in published maps and institutional affiliations.

Ready to submit your research? Choose BMC and benefit from:

- fast, convenient online submission

- thorough peer review by experienced researchers in your field

- rapid publication on acceptance

- support for research data, including large and complex data types

- gold Open Access which fosters wider collaboration and increased citations

- maximum visibility for your research: over $100 \mathrm{M}$ website views per year

At $\mathrm{BMC}$, research is always in progress.

Learn more biomedcentral.com/submissions 